\title{
Implicit Error, Uncertainty and Confidence in Visualization: an Archaeological Case Study
}

\author{
Georgia Panagiotidou, Ralf Vandam, Jeroen Poblome, and Andrew Vande Moere
}

\begin{abstract}
While we know that the visualization of quantifiable uncertainty impacts the confidence in insights, little is known about whether the same is true for uncertainty that originates from aspects so inherent to the data that they can only be accounted for qualitatively. Being embedded within an archaeological project, we realized how assessing such qualitative uncertainty is crucial in gaining a holistic and accurate understanding of regional spatio-temporal patterns of human settlements over millennia. We therefore investigated the impact of visualizing qualitative implicit errors on the sense-making process via a probe that deliberately represented three distinct implicit errors, i.e. differing collection methods, subjectivity of data interpretations and assumptions on temporal continuity. By analyzing the interactions of 14 archaeologists with different levels of domain expertise, we discovered that novices became more actively aware of typically overlooked data issues and domain experts became more confident of the visualization itself. We observed how participants quoted social factors to alleviate some uncertainty, while in order to minimize it they requested additional contextual breadth or depth of the data. While our visualization did not alleviate all uncertainty, we recognized how it sparked reflective meta-insights regarding methodological directions of the data. We believe our findings inform future visualizations on how to handle the complexity of implicit errors for a range of user typologies and for highly data-critical application domains such as the digital humanities.
\end{abstract}

Index Terms - data uncertainty, data visualization, implicit error, qualitative study, digital humanities, design study, archaeology

\section{INTRODUCTION}

A RCHAEOLOGY studies the historical trajectories of past communities by collecting, measuring, classifying and interpreting past material cultures. As the understanding of past artifacts and their original contexts of use is inherently incomplete, archaeological researchers typically collect data through a wide variety of means so they can more confidently ground their interpretations. The synthesis of different types of discrepant data together with tacit and experiential knowledge forms a core part of modern archaeological reasoning [1]. However, as archaeological reasoning is an evidence-based process, the representational choices of data and artifacts can significantly skew the interpretations being made [2], [3]. Accounting for data uncertainties in visualizations is therefore a crucial challenge in archaeology as in other humanities disciplines [4], [5], |6].

Research in information visualization identified different types and origins of data uncertainty [7], [8], |9], |10], among which those originating from implicit error. Implicit error is a type of measurement error that is inherent to a dataset but is not explicitly recorded, so that experts can only account for it in qualitative ways based on their implicit domain knowledge |11|. In field work, for example, situational circumstances such as weather and local conditions can produce such errors. Embedded within a long-term archaeological research project that is attempting to embrace more data-driven and computational research

- G. Panagiotidou is with R[x]D and the Sagalassos Archaeological Research Project, KU Leuven. E-mail: georgia.panagiotidou@kuleuven.be

- R. Vandam is with the Sagalassos Archaeological Research Project, KU Leuven and the Vrije Universiteit Brussel.

- J. Poblome is with the Sagalassos Archaeological Research Project, KU Leuven.

- $\quad$. Vande Moere is with $R[x] D, K U$ Leuven. methods, we encountered the impediments of implicit error ourselves. Three implicit errors emerged when archaeologists interacted with our preliminary visualizations of a large collection of spatio-temporal archaeological site data, causing them to be critical towards the data rather than to form useful insights. These errors were related to differing collection methods, the subjectivity of data interpretations and to assumptions of temporal continuity.

As it is known that sense-making is a calibration process between the user, the representation and the data |9], [12], we wanted to uncover how explicit representations of implicit error alongside the data impacts this process. Following recent calls for rigorous research that considers the human side of data science [13], we chose to capture this impact by developing a visualization 'technology probe' |14 that exemplified a potential representation of the implicit errors. In our study design, we used this probe to capture in-depth qualitative and quantitative data data on how 14 archaeologists, grouped by their levels of archaeological domain expertise and relation with the underlying data, dealt with uncertainty originating from implicit errors. This study thus investigates: (RQ1) how the visualization of implicit error relates to uncertainty and confidence in the generated insights; (RQ2) how the visualization features can be appropriated to cope with implicit error; and (RQ3) how the background of the user influences these appropriations.

We found that including implicit error in archaeological settings helped domain experts be less critical of the visualization tool overall even if they did not use the dedicated features, while data novices became actively aware of the implicit errors in the first place. We observed that visualizing implicit error sparked reflective meta-insights regarding operational aspects of the archaeological research. Still, the 
visualization in itself did not alleviate all origins of uncertainty in the data and we observed how participants quoted social factors to ignore some, while in order to minimize others they requested additional contextual breadth or depth of the data. Reflecting on our role as immersed researchers in this process, we note how implicit errors became more clearly articulated when nudging our collaborators beyond their current data practices. Although originating from an archaeological case study, we synthesize our findings into takeaways that can inform future research on how to elicit, visualize and support coping with implicit error in the analysis proces: 1

\section{Related Work}

\subsection{Data Uncertainty and Trust}

While research on uncertainty in visualization often revolves around quantitative presentations of error, an increasing body of work examined uncertainty visualization in its broader settings of supporting analytical reasoning and decision-making [8], [12], [15], [16], [17], [18]. Kim et al. [19] illustrate how decision-making with visualizations follows a Bayesian cognition model and show that a reader's posterior decision to trust the findings, is dependent on their prior beliefs as well as to the visualization shown. Sacha et al. [9] modeled nine different types of uncertainty and their propagation over a human analytic process with visualizations, and demonstrated how users' awareness of potential uncertainties impacts their trust in visualization systems more broadly. Still, some data uncertainties relate to the collection, digitization and original interpretation of data sources [20] and accordingly originate from even earlier stages of prototypical visual analytics systems and are thus often excluded from them. Similarly, uncertainty originating from implicit error is closely dependent to the knowledge and experiences of the analyzing domain experts and is thus hard to capture and explicitly encode [11].

Without awareness of the underlying uncertainty in an information system, analysts are not able to accurately assess and trust findings emerging from it [9]. As such, an individual's domain expertise, previous experiential knowledge [21], and their clarity on why they are certain or uncertain towards a statement in the first place [22] impacts their handling of uncertainty as they relate to such awareness. This is also the case for data visualization systems, as the lack of domain expertise is shown to impact the overall attentiveness of one self's mental reasoning processes [23] and thus by extension their awareness of uncertainty.

Discussions in archaeology [2] and other humanities [24], [25] state that any reconstruction developed for reasoning, whether an interactive visualization or other, is in itself a 'reasoning artifact' [26] that enables progressive theory building - i.e an evolving understanding of a phenomenon. Accordingly, archaeological research discusses uncertainty in relation to bias and reasoning [1], [27], and argues that many types of uncertainty tend to be subconsciously propagated into theoretical or visual reconstructions of the past [28]. The most common type of uncertainties that need to be managed in archaeology are epistemic in nature [29],

1. Link to supplementary material: https://osf.io/th2ck as they result from having incomplete or only partially available data. Therefore awareness of the progressive loss of information throughout data analysis is important in the field [30| while reductionist attitudes are occasionally greeted with skepticism [31].

\subsection{Representing Uncertainty}

A strategy tested in multiple visualization contexts (e.g. casual, visual analytics, communication) is visualizing uncertainty as an additional dimension of the data. This uncertainty dimension can then be encoded as fuzziness, opacity, sketchiness, coincidentally or adjacently among many others ( [6], [32], [33], [34] provide extensive documentation of such uncertainty encodings). Despite such research, uncertainty is often not included in visualization-driven communication however, as it is perceived to add challenges [35]. Visualization authors feel that encoding uncertainty might obfuscate the visualization 'message' for the readers while they also express a lack of standardized methods to visualize uncertainty without encoding it 'imprecisely'.

Nevertheless, research shows additional ways to inspire trust in the data besides visually encoding uncertainty as a separate dimension. Greis et al. [36] demonstrated how systems that provide access to primary sources of information inspire more trust than those which only show aggregated values. Recent studies that examined uncertainties during decision-making in a research workflow similarly suggest that codifying and visualizing progressive decision points helped support transparency and reflection over the process [17], [37]. Working with implicit errors, McCurdy et al. |11| illustrate how researchers can textually capture and then show the descriptions of implicit error alongside visualization to inform the analytical sense-making process. Following a different approach, Franke et al. [20] quantified the confidence in the data, rather than its uncertainty, and visualized it as a hierarchical color-coded tree alongside other dimensions.

The digital humanities discipline, which includes the field of archaeology, has argued that its users prefer rich, direct and multi-faceted representations [38], [39], [40], [41], [42] above traditional, reductionist visualizations. As such, visualizations for digital humanities experimented with including imagery of the original objects [38], [41], [43], digitally simulating piles and streams of tangible artifacts [43], including multiple entry points as a way to spark curiosity [38], or by adding a 'beautiful mess' as a way to support ambiguity of representation during analysis [44]. While Theron et al. [29] suggest data provenance and computationally accounting for propagated uncertainties can be used to inspire confidence in the humanists conducting visual analysis.

In terms of evaluation, uncertainty in visualization is analyzed in various casual situations, including weather forecasting [36], [45] and commuting timetables [16], [46] and under multiple different scenarios of use, including incentivised simulations [16], controlled tasks [45] or inthe-wild [36]. Yet most commonly, uncertainty visualization is evaluated in relation to assessing a design technique rather than focusing on the interpretational aspects of its impact [47]. Two recent notable studies that, similar to this 
work, deployed rigorous design-oriented research and indepth qualitative user studies to study the situated nature of uncertainty during data analysis in specific domains include McCurdy et al. [11] and Kale et al. [15]. Moreover, this work presents a qualitative study in humanistic research settings, a field that has documented data and visualization issues, yet lacks a closer examination of their relation to uncertainty and sense-making.

\subsection{Terminology}

For clarity, we summarize our use of the terms uncertainty, confidence and implicit error throughout this manuscript. In persuasion literature, certainty describes a persons' belief that an attitude they hold is valid [21] while in data visualization studies the term uncertainty often is used to refer to uncertainties of the data i.e. the discrepancy between a data measurement and the phenomenon of study (e.g. [9], [48|). We use the term uncertainty similarly to [10], [15], to denote moments of doubt when sense-making with data which can therefore include aspects that go beyond the data itself. We operationalize uncertainty by capturing confidence in findings or statements as previously documented [47], [49] and utilized [18], [48] in visualization research. Therefore, implicit errors are discrepancies that, just like errors that appear while data modeling, are sources of uncertainty.

\section{The Case Study}

This research took place as part of the long-standing and internationally known Sagalassos Archaeological Research Project. Located in south-west Turkey, the archaeological site of Sagalassos has been the focus of intensive interdisciplinary research for over thirty years. Though originally approached for its uniquely preserved ancient urban site remains, researchers expanded their focus to better understand the long-term development of socio-ecological systems in the study region of ancient Sagalassos. In their examination they hope to address a multitude of archaeological research questions, including: Why were settlements built at specific locations? What drove their continuity or instability over time? How did the countryside change throughout the rise and decline of Sagalassos? The archaeologists approach these questions through a collection of site data along the region that indicated periods of human activity, with a site referring to a single location. This spatiotemporal dataset included samples from almost 300 locations and 15 eras.

During a one-year 'design by immersion' [50] collaboration, the first author investigated how these archaeologists collected, documented and represented their data and cautiously made interpretations. In practice, the first author worked on the project premises on a weekly basis, conducted preliminary workshops with archaeologists, as well as accompanied them during their field-work for two weeks. These interactions gave her the opportunity to formally and informally observe and interview domain experts of varying levels of expertise from undergraduate students and excavation supervisors to established academics. We adopted a distributed cognition understanding [51], in which the archaeologists' reasoning was examined as a situated experience that is formed through the interaction with external representations and thus paid close attention to their use of visualizations. We noticed that a considerable amount of available site data was still largely unexplored in their temporal dimensions mostly due to a lack of dedicated means to effectively query, cluster and explore the various site parameters. We therefore collaborated more closely with three of the more senior archaeologists to create such a tool to be used by the archaeological team at large.

Inspired by the attribute-signatures design of Turkay et al. [52], we shared a conceptual approach for using 'timeprints', as small recognizable sparklines of each site that embodied the archaeological concept of continuity and instability over time (see Figure 1-b). These 'time-prints' were met with excitement by our three close collaborators for their ability to visually represent the theoretical construct of instability as well as their improved analytical power compared to the current practice of using 'dots-on-map' (Figure 1 -a). This initial excitement however, gave way to a critical disbelief towards the findings that emerged from these time-prints, which seemed to be caused by the presence of three implicit errors. Accordingly, our next iteration included dedicated features to address these implicit errors.

We used this iterated visualization as a technology probe [14] i.e. a functional prototype, deployed in real settings, to query users' attitudes towards a technologicallyoriented phenomena. Unlike a prototype, which is deployed in order to get feedback and be improved upon, a probe may end up a stepping stone for a different design entirely. So while our visualization probe was designed based on specific domain requirements, its core purpose was to elicit reactions on the handling of implicit errors and uncertainty from their visualization. (a)
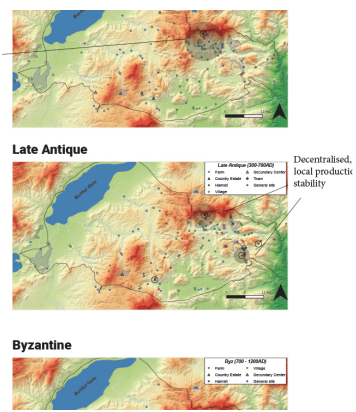

(b) Site Time-prints

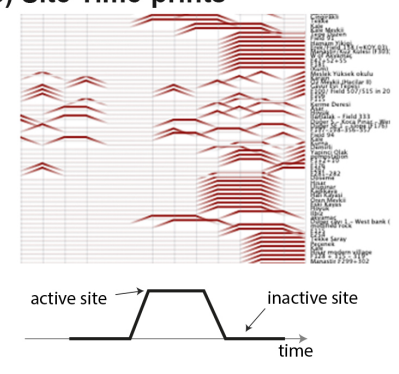

Fig. 1. (a) The current practice of 'dots-on-map' used for showing site patterns. Each map represents one time-period (era). (b) Our first redesign for a temporal view of sites as 'time-prints' where each row represents a site in the region oscillating depending on if it was active or inactive over time. This representation was originally approached with enthusiasm because it helped uncover clusters of similarly unstable sites but was later critiqued for its implicit errors.

\subsection{The Visualization Probe}

The visualization probe included basic features to support insight-making as well as dedicated features to address the implicit errors. The choice of basic features was driven by existing practice in spatio-temporal data visualization and visualization practices in the humanities. Specifically, it was divided in three main sections connected through brushing 
and linking, each revealing a temporal (Figure 2), spatial and summary perspective of the data.

In the temporal section, each site, accompanied by its name, was depicted as a row in a matrix. The columns of the same matrix represented time in eras. If a site was active during a specific era, it would be indicated in the matrix as a point, a line or a colored rectangle depending on conditions described further on. The sites in the matrix could be sorted by different criteria, such as the site total activity over time, its instability or the year of the data collection among others. The spatial section depicted the sites on a geographical map. Sites were selectable individually or in groups from the map, the matrix (through brushing) or could be queried directly by their name or region. Once selected, additional information such as any field notes and other metadata appeared on the right in the summary column.

To provide domain experts both an overview as well as details-on-demand for each individual site - or to support distant and close reading as it is known in the humanities [53] - we included a condensed and an expanded zoom level of the temporal section (Figure 2 $\mathrm{E}$ ). A video demonstration can be found in the supplementary material.

\subsection{Emerging Implicit Errors}

The dedicated probe features for the three emerging implicit errors resulted after discussions with our close collaborators. For each error, we describe the contextual situation of how the error manifested, followed by the dedicated features in the visualization probe that address it.

\subsubsection{Differing Collection Methods (U1)}

Excavation, arguably the most known archaeological data collection method to date, forms a tedious, intrusive and relatively spatially-limited research activity, and is therefore deployed in very promising settings. Archaeologists prefer to use a combination of alternative data collection methods together with excavation, such as field surveying [54|. Our collaborators commonly use field-walking, a nonintrusive field surveying method during which a team of archaeologists explores and records, either systematically or not, the landscape on the ground for archaeological surface materials. Typical surface materials range from lithics to architectural remains, and from singular standing structures to numerous pottery sherds. Unlike excavations, which provide extremely detailed and well-preserved information on a small physical area, surface surveying paints broader pictures of archaeological landscapes through the existence or absence, of archaeological sites and foci through time.

Within the study region, only nine sites were (partly) excavated, whereas more than 270 sites were surveyed by two types of field-surveying methods [55]. During the first years, the project deployed 'extensive' surveys namely nonsystematic visits along fields, often based on tips from locals. In more recent years, the group turned to 'intensive' surveying methods, i.e. the systematic division of an area of interest in plots on which archaeologists walk and collect finds along predefined transects or line grids. The discrepancies between the two survey methods makes comparing older and newer archaeological finds challenging and prone to error due to differences in sample size, spatial resolution and the types of the finds (e.g. prominent monumental fragments versus finer lithics). Moreover, contextual information of each surveyed area, such as the ground visibility or landscape features remains tacit to those archaeologists who actually conducted the surveying. In the original site dataset, these rich implicit errors were reduced into a single item row describing an area (by its ID) and a corresponding indication for the existence or absence of a site over time.

Dedicated Probe Features. In order to more transparently denote how the data was collected (U1), we externalized the three deployed data collection methods, i.e. the excavation and the two survey types, as an additional dimension. Practically, these methods were encoded as a background pattern on each row in the matrix (Figure 2-C). The background patterns were of progressive visual density so as to mirror the density and cover of the collection methods. This data methods view, as we termed it, could be shown or hidden at any instance. As the origin of this error is of an ordinal dimension we chose a coincident (i.e. showing data and uncertainty integrated in the same display) and extrinsic (i.e. using an additional graphical object) techniques [34], to externalize it in the probe. Extrinsic representation of uncertainty seems to outperform intrinsic ones (e.g. using opacity or color saturation) especially in cases of communicating qualitative uncertainty to experts [34]. Accordingly, coincident approaches are preferred in cases of low visual clutter as they require less effort to relate data and their uncertainty [34].

The approximate coverage of each collection method was explicitly described in the legend (Figure 2fF). While the numerical percentages of coverage mentioned in the legend were imprecise, they were nevertheless included to highlight the differences between the three data collection methods for users who lacked that previous knowledge.

\subsubsection{Subjectivity of Interpretations (U2)}

Collected archaeological finds are studied by material specialists who date and interpret their nature and patterning. Ceramologists, for instance, look at a range of characteristics such as the fabric (texture, color, inclusions) and morphology of pottery sherds as their features provide crucial information on provenance, function and chronology [56]. It is commonly shared knowledge within archaeology however, that the experience and overall exposure of ceramologists to location-specific fabrics and styles influences their classifications. Therefore, subjectivity and bias can creep into classifications over different individuals as well as over time as ceramologists hone their skills.

Once all artifacts are dated and classified, archaeologists interpret their amount, type and distribution to label the sampled area as a site. Furthermore, using theoretical models, they then further interpret those sites as farms, hamlets, towns, production sites or necropoleis based on the amount, nature, function and distribution between the finds, the existence of monumentality or other special types of materials (e.g. coins). These interpretations can differ between individual archaeologists depending on their assumed theoretical stance, adding another layer of implicit error to the source data. The site dataset we worked with did not directly capture such theoretical stances or the subjective 


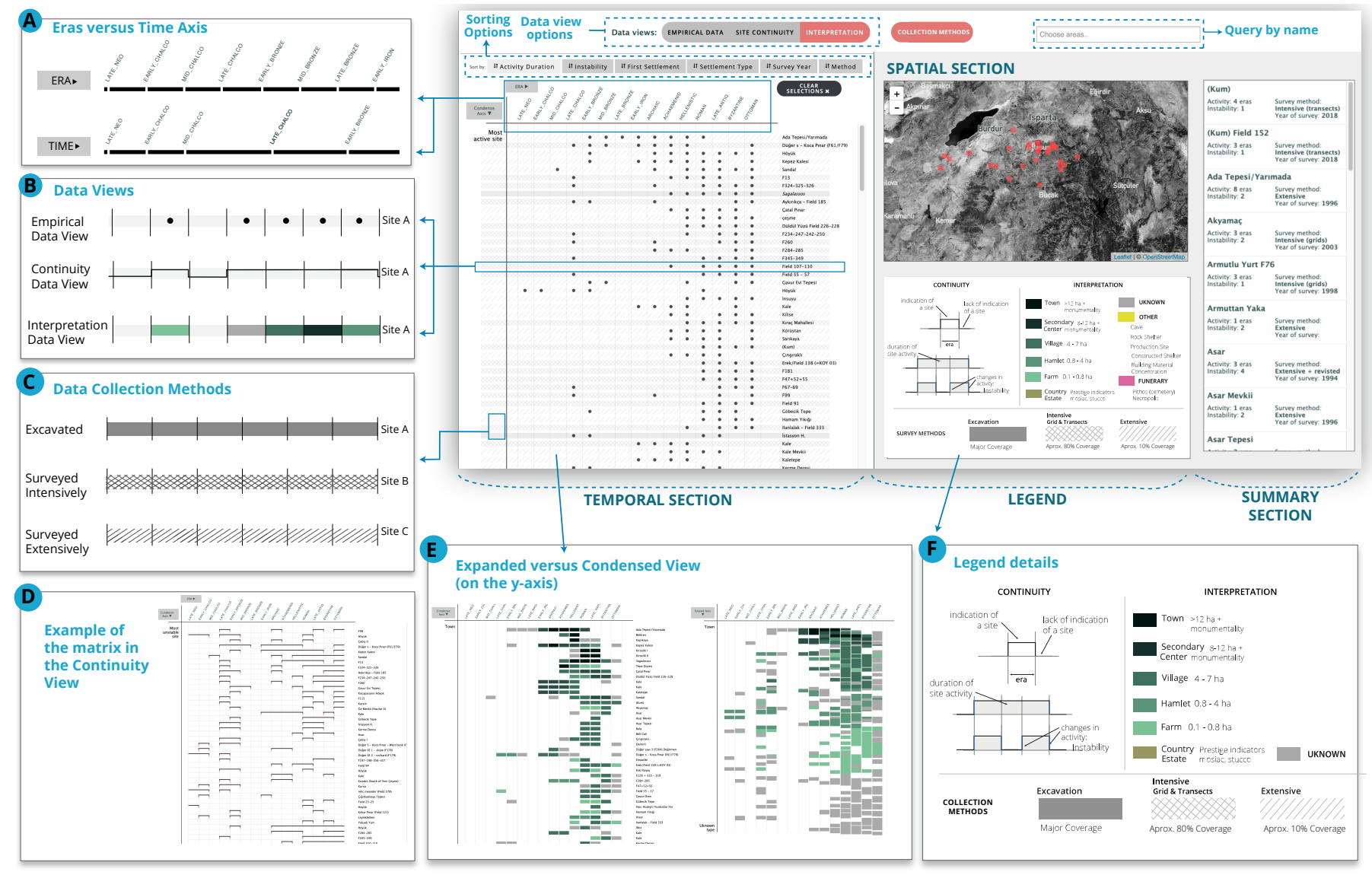

Fig. 2. Screenshot of the visualization probe with its main sections showing temporal, spatial, summary perspectives as well as the legend. The matrix in the temporal section shows each site as a row with the columns representing time. $(A)$ depicts the axis variation in chronology (eras) or in absolute years. Together with the continuity data view shown in (B) these features are meant to address implicit error U3. (B) depicts the different representations a site can have in the matrix: empirical, continuity and interpretation. Each view progressively encodes assumptions that can impact the findings. This separation of views was included to bring awareness to implicit error U2 and U3. (C) depicts the encoding of the data collection methods - implicit error U1- as a background pattern in each site/row. (D) depicts the continuity data view with sites here ordered from most to least stable in the region. (E) illustrates the basic feature of detailed and distant reading when using the interpretation data view. In this specific instance the distant reading clearly shows how farms and hamlets (light green) mostly appear after Hellenistic times (i.e. closer to the right of the $y$-axis). $(F)$ depicts details from the legend including how we quantified instability of sites, the classification parameters of the site types and the collection methods.

interpretations that went into classifying each site as e.g. a 'town', 'hamlet' or 'unknown'.

Dedicated Probe Features. To account for the implicit error brought by such interpretation subjectivity (U2), the probe included interpreted (interpretation) and noninterpreted (continuity and empirical) data views that represented the data on two different semantic levels.

The interpretation view represented the site types (e.g. hamlet, village) and color-coded them based on the adopted theoretical classification model from one of the close collaborators. In this case an active site in a specific era was represented in the matrix as a colored rectangle indicating its type (Figure 2-B \& E). The chosen color scheme was parallel to the classification wherein 'hamlets', being the smallest settlements were represented in lighter and 'villages' and 'towns' were represented as progressively darker green (reduced in luminosity while maintaining hue). At the same time, the visualization legend (Figure $2-\mathrm{F}$ ) clarified that the classification model was based on the relative sherd distribution area per hectare.

Contrary to the interpretation view, the empirical and continuity views only visualized the site existence and did not encode the site types. Visually, that meant they were uniformly colored in black. Still, the continuity view represented the site as an oscillating line over time, whereas the empirical represented each site as discrete points among eras, for reasons discussed below (U3).

As the subjective and interpretive errors of $\mathrm{U} 2$ are hard to quantify, we instead opted to bring awareness on the data interpretation pipeline and accordingly on the error propagation that came along with it (similar to [17], [37|). Our hypothesis being that the views would be used depending on the level of data assumptions researchers were comfortable with adopting (no assumptions, adoption of time continuity (U3), adoption of subjective interpretations (U2)). By using three separate data views users can explore the effects of different assumptions separately and on demand, in fact following a dynamic and adjacent approach to uncertainty visualization [34]. 


\subsubsection{Continuity with Discrete Data (U3)}

A core practice in archaeology is estimating a pattern of temporal continuity for a site. Nevertheless, the dating techniques classified finds in eras rather than years meaning that such continuity is merely an assumption. The presence of artifacts from a particular time period does not necessarily entail that a site was actively and continuously used throughout a whole era. For instance, while uncovering lithics in a location may classify its surroundings as an active site in Epipalaeolithic times (i.e. the last phase of the Old Stone Age), the Epipalaeolithic era spans over 1,000 years making it impossible to know if it was active throughout all that time. Moreover, as eras are culturally defined, they vary in terms of duration (from hundreds to thousands of years), so assumptions of continuity also depend on the specific era being discussed. Perhaps because archaeological data is rarely continuous, we found that our collaborators still wanted to hypothesize about patterns of continuity, as long as they could be made aware of how estimations of continuity carries along these implicit errors.

Dedicated Probe Features. To represent the implicit error that comes with assuming site continuity (U3) we added two dedicated probe features. First, in the continuity view we represented the site as binary signals over time as step-graphs (see Figure 2 $\mathrm{B}$ \& D); while also offering the non-continuous version of the same information in the empirical view. An active site during an era was then either represented as a line or a point, breaking the visual correlation to continuity as needed. Secondly, we highlighted the duration differences among the eras, by designing the visualization temporal axis to switch scale among absolute years and equidistant chronology in eras (Figure 2-A).

\section{Study Design}

Although forming a convenience sample, we aimed for a sufficiently stratified participant cohort. As such, we recruited a total of 14 participants ( 9 male, 6 female) which were specifically chosen to possess varying levels of experience with both archaeological research and data fieldwork (see Table 1 for a detailed break-down). When recruiting experts we consciously chose participants that had some tangible relation to the region of study. While this may seem counter-intuitive when wanting to generalize the conclusions, it nevertheless reflects the realities of archaeological research practice in which researchers typically specialize in a single region and/or a cultural period.

The recruitment call invited participants to test a visualization of the Sagalassos site data without mentioning the focus of the study to capture the effect of including implicit error in the visualization. All but two participants (P8, P6) had not seen or interacted with the visualization probe beforehand. Although these two participants had been part of the initial collaboration process, this study formed the first opportunity to actively use it to create insights.

The participants were classified based on their current level of archaeological domain knowledge, i.e. domain novices who were educated as archaeologists (i.e. BA and MA students, $n=7$ ), and domain experts who have actively engaged in archaeological research (i.e. PhD and Post-Doc researchers, $\mathrm{n}=7$ ). As students actively participate in archaeological fieldwork, it is not uncommon for domain novices to have first-hand experiential knowledge of specific field settings of survey data collection while a domain expert may not. We thus defined two levels of experiential data knowledge: data novices $(\mathrm{n}=6)$ had no previous first-hand relation to this or any survey data; data experienced participants $(n=8)$ had first-hand experience collecting data through archaeological surveying during at least two occasions.

\subsection{Setup and Tasks}

Participants were interviewed either in dedicated professional premises or via video-conferencing, depending on their availability. Each session started with an identical demonstration of all available visualization features (taking approximately 6 minutes). With the prior explicit permission of the participants, each session was digitally logged and recorded via audio (in-person sessions) or audiovisual (video-conferences) media. The study involved three types of tasks, which were specifically designed to capture wellrounded and comparable empirical data, and were refined through a prior pilot test with two representative users that were excluded from the final sample.

Exploratory Analysis. As a first task, each participant was asked to use the probe to find interesting patterns, observations or outliers. Each insight had to be captured as a screenshot which could be annotated by short descriptive notes. After 15 minutes of uninterrupted exploring, and by using each screenshot as a memory aid, each insight was discussed with the interviewer to uncover the motivation, process, confidence, novelty and perceived data quality. Due to the specificity of the video-conferencing medium, the insight-making process was discussed aloud by the participants while it was happening. Accordingly, during these sessions the exploratory task was given an additional $15^{\prime}$ of time (for a total of $30^{\prime}$ ) to accommodate for the additional cognitive effort of exploring while thinking-aloud [57].

Site Comparison. For the second task, participants were briefed that a colleague believed that two archaeological sites (A and B) seemed to be forming a cluster. They were asked to use the probe to compare the two sites and form an opinion on what extent they agreed or not with that claim, all while externalizing their reasoning following the think-aloud protocol. The task was specifically chosen to include two sites with highly similar temporal development patterns (both appearing during Roman and continuing until Ottoman times), yet differing only in terms of implicit errors U1 and U2. First, they contrasted in terms of survey methods (U1), i.e. A being extensively versus B intensively surveyed; and in terms of their classification (U2), i.e. A 'peaking' as a hamlet, whereas B developed into a village.

Closing Interviews. At the end of each session, each participant was asked to assess which of the probe features they used more and why. They were also asked to self-assess their saturation of findings (i.e. to what extent they felt they had explored the visualization for findings) on a scale of 1-100 explaining their reasoning, and lastly, to note three aspects they liked most and the three that needed improvement or were missing in the visualization. We used the metric of saturation of insights after a set time of exploration, as 
TABLE 1

Recruited participants ordered left to right by domain experience. (C): indicates that participants were part of the data collection, or (C\&I): both data collection and interpretation. $\left(^{*}\right)$ indicates as being part only of basic training.

\begin{tabular}{|c|c|c|c|c|c|c|c|}
\hline ID & $\begin{array}{l}\text { Domain } \\
\text { Novices }\end{array}$ & Relation to Data & Software Skills & ID & $\begin{array}{l}\text { Domain } \\
\text { Experts }\end{array}$ & Relation to Data & Software Skills \\
\hline 5 & BA & novice & Excel & 1 & $\mathrm{PhD}$ & novice & Google Earth, Databases* \\
\hline 10 & BA & experienced (C) & GIS* & 6 & $\mathrm{PhD}$ & experienced $(C \& I)$ & GIS, Excel \\
\hline 7 & MA & novice & GIS* & 13 & $\mathrm{PhD}$ & experienced $(C)$ & Excel \\
\hline 4 & MA & novice & Scripting* Databases & 2 & Post-Doc & novice & GIS, Excel \\
\hline 14 & MA & novice & GIS*, Scripting* & 3 & Post-Doc & experienced $(\mathrm{C} \& \mathrm{I})$ & GIS, Excel \\
\hline 11 & MA & experienced $(C)$ & GIS, $R^{*}$ & 8 & Post-Doc & experienced $(\mathrm{C} \& \mathrm{I})$ & GIS, Excel \\
\hline 12 & MA & experienced (C) & GIS*, GoogleEarth & 9 & Post-Doc & experienced (C\&I) & GIS, Excel \\
\hline
\end{tabular}

previously used by Saraiya et al. [58|, to understand the perceived richness of the visualization for making insights.

\subsection{Data Collection and Analysis}

To capture the confidence in the findings (RQ1), we mapped the type and amount of uncertainties participants encountered in their insight-making process by examining the session transcriptions. Together with the digital logs, we then compared if and how the probe helped elicit this confidence (RQ2). Finally, we used the comparison task to understand how the uncertainties were acknowledged and managed by the different participant types (RQ3).

\subsubsection{Capturing Confidence in the Findings}

We recorded 63 insight-making cycles [59] guided by the participant screenshots. Insight 'units' were self-reported by participants. While each screenshot was originally expected to represent a single insight, participants often used the same screenshot to indicate two or more.

The transcribed insight-making cycles were analyzed through two rounds of progressive thematic analysis informed by existing codes [60|. Following previous evaluation strategies of uncertainty in data visualizations [47], [48], [49], we operationalised uncertainty as lack of confidence. In the first coding round, within each transcribed insight we identified moments in which the participants - either when prompted or in their own accord discussed or indicated confidence or doubt regarding their observations, their hypotheses, the data or the visualization. We looked for indications of confidence both expressed directly e.g."I'm not confident.., 'I don't know..'" as well as through indirect and contextual wording e.g. " $I$ ' $m$ not a specialist but..", "This struck me.."). We interpret these moments to be similar to the uncertainty instances described in [10|'s coding scheme of data workers' task process as well as moments of uncertainty in decision-making with data as described by [15]: all describe situations of uncertainty during the analysis, even if in our case these situations are more nuanced as originating from the micro-scale of a visual analysis process rather than a macro-scale of a complete data analysis pipeline.

In the second round of coding we focused exclusively on those moments after removing any repetitions of the same uncertainty in a single insight-cycle (e.g. because of participants' verbal repetition). We then grouped them based on the origins of uncertainty (including the original three implicit errors). The grouping was initially conducted through open coding with the resulting codes being then progressively abstracted into larger themes with the aid of codes from existing literature that describe uncertainty as arising from data, model, interface and cognitive sources [10]. Through this process, six sources of uncertainty were uncovered. Lastly, and when possible, the participant's coping strategies, i.e. ignore, understand or minimize |10] were also identified for each moment.

Both rounds of coding were conducted by the first author yet partial results and issues were discussed with the last co-author throughout. The output of the thematic analysis is a code-book that collects the final definitions of the six appearing themes as well as indicative quotes from the transcripts that was also later used to analyze the transcripts of task 2. We share this code-book as supplementary material for transparency but also in the hope that it can aid the comparison of emerging results from future case studies.

\subsubsection{Assessing the Role of the Probe Features}

The digital logs from the sessions were analyzed separately by documenting the absolute and relative time each participant spent on each probe feature as well as the flow between them. This quantitative data was cross-referenced with the participant statements from the closing interviews regarding their feature preference as well as moments during the insight-making in which explicit mentions to probe features were made. Accordingly, they helped clarify if and how any expressed uncertainty was prompted by the probe.

\subsubsection{Comparing Participants Types}

Since all participants shared this task, we used the comparison task to analyze how uncertainty coping mechanisms were deployed depending on their background. This task was similarly analyzed through the transcription of the participant's sense-making process. The transcriptions were examined to see if and how participants recognized the sources of uncertainty (U1, U2) and how they coped with them, while we also examined them for instances of other coded uncertainties from the code-book.

\section{Results}

Each of the 14 interviews lasted between $1 \mathrm{~h}-1.5 \mathrm{~h}$ hours, resulting in $15 \mathrm{~h} 47 \mathrm{~m}$ of recorded data, of which $10 \mathrm{~h} 05 \mathrm{~m}$ was transcribed and complimented with an additional $5 \mathrm{~h} 25 \mathrm{~m}$ of interaction logs.

Exploratory Analysis. All participants went through three to nine insight cycles, many of which reached beyond 
TABLE 2

Sources of Uncertainty

\begin{tabular}{|c|c|c|}
\hline & Uncertainty Sources & Example Quotes \\
\hline $\mathrm{U} 1$ & Data Collection & $\begin{array}{l}\text { "Well at least here it is intensive survey so there are some good indications [..]"(P2), "I don't know if that is a question of research methods or } \\
\text { if it is a question of what the actual history of the territory is [..]" (P6) }\end{array}$ \\
\hline $\mathrm{U} 2$ & Data Interpretation & $\begin{array}{l}\text { "I do not think it might have been a Town if it was Unknown because monumentality is pretty conspicuous. It would be hard to overlook that." } \\
\text { (P1). "[..] I would be more confident if I would know the unknown data is a farm or something else"(P5). }\end{array}$ \\
\hline U3 & $\begin{array}{l}\text { Temporal \& Spatial Rep- } \\
\text { resentation }\end{array}$ & $\begin{array}{l}\text { "Of course these are still long periods [..], even if there is only one little block [..]" (P1). "It is not 100\% there but close to or in the region of } \\
\text { those settlements (pointing to the map)"(P14). }\end{array}$ \\
\hline $\mathrm{U} 4$ & Vis. Development & $\begin{array}{l}\text { "The starting point of this visualization is of course that I trust you to have used the right data."(P1), "remember there were a lot of artifacts } \\
\text { just labeled pre-historical. How did you handle these situations?"(P9). }\end{array}$ \\
\hline U5 & Hypothesis/Observation & $\begin{array}{l}\text { "So it's a very normal pattern the interesting thing is that there are some other examples"(P2),"That is something that struck me.". "..,that } \\
\text { seemed to me a bit odd", "Well I feel like there should have been more farms or hamlets"(P13). }\end{array}$ \\
\hline U6 & Background Knowledge & $\begin{array}{l}\text { "[..] I am not a survey specialist." (P2). "I know that because I have experience of working in there"(P8), "[..] but I do not know the [location] } \\
\text { material that well."(P9). }\end{array}$ \\
\hline
\end{tabular}

simple observations. Participants familiar with the data created at least one original hypothesis, often directly related to an implicit error. We separated a hypothesis from an observation following the definitions by [58], with hypotheses having potential to evolve into wider research agendas. When asked about the saturation of findings all participants claimed that in their time of use (15' or $30^{\prime}$ minutes) they uncovered less than half $(50 \%)$ of what the probe could permit, giving us a good indication of the probe's perceived utility and completeness.

Participants started their insight-making process either by testing hypotheses or by openly exploring. Domain novices, who for the most part were openly exploring (5 out of 7), used the expanded view of the data and iterated through the probe features in rapid cycles. Domain experts on the other hand seemingly explored the probe's features as much as the data, as they typically used each data view for longer; sorted sites through the predefined options; and flowed between the spatial and temporal dimensions to make findings.

A subset of these insight cycles, which we refer to as reflective meta-insights, indicated with a star symbol in Figure 3. resulted directly from externalizing the first implicit error (U1) in the visualization probe. Specifically, during these insight-cycles, participants investigated the data collection methods as a unique data dimension instead of using it for contextualizing the main dataset. Through these reflective meta-insights, participants with first-hand experience in collecting data made hypotheses directly relating to the methodological and operational aspects of their research which drove them even to consider their choices for future surveys. "Extensive survey seems to have a similar distribution of site types [as in intensive survey], there are still towns, secondary centers, villages, hamlets"(P13). Here P13 is describing their surprise that even though extensive is less systematic than intensive surveying, it equally managed to uncover even the smaller scale settlements such as villages and hamlets, thus making her reflect on future deployment methods.

Site Comparison. The comparison task lasted approximately 5-6 minutes, during which participants typically searched for the two regions of interest and then progressively switched through the data views, including the map. All participants identified the similarities (same occupation duration and development peak) and all but one (P10) identified the differences in survey method and classification.
While participants used the probe in comparable ways and responded that the sites in question were indeed closely related, this argument was supported by different depths of reasoning and analytical evidence.

\subsection{Moments of Uncertainty}

From the 63 insight-making cycles, 61 included at least one moment of uncertainty leading to 130 unique uncertainty moments. Once classified, these uncertainties appeared to be originating from six sources, in fact three more origins than the implicit errors the visualization probe was purposefully designed for (see also Table 2). These include:

U1- Uncertainties from Data Collection Methods are moments during which the participants questioned how the data was collected. These were the second most encountered source of uncertainty (27 from 130) as all participants vocalized how the data collection methods impacted their confidence in a finding. While this implicit error was purposefully designed for in the probe, these moments appeared in slightly broader expressions than how we initially expected, such as contextual concerns about specific sites, dates or research bias in general.

U2- Uncertainties from Data Interpretation are moments during which the participants expressed concern about how the data was classified or interpreted. As this uncertainty was also purposefully designed for in the probe, it included participants' issues with the site classification system, its theoretical underpinnings, as well as its application on this data. For instance "I think we should take these settlement-type / size-metrics with a grain of salt" (P6). This source of uncertainty occurred in 23 out of 130 instances.

U3- Uncertainties of Temporal and Spatial Representation are moments in which the participants questioned how the data was visually presented. Although we originally designed for this implicit error considering only its temporal dimension and the issues of representing discrete data as continuous, we also encountered it relating to spatial imprecision related to the map section. This type of uncertainty occurred infrequently (8) regardless of participant background.

U4- Uncertainties from Visualization Development are concerns relating to how the data was modified, adapted or further classified for the visualization. Although they were mentioned only once or twice per participant rather than per 


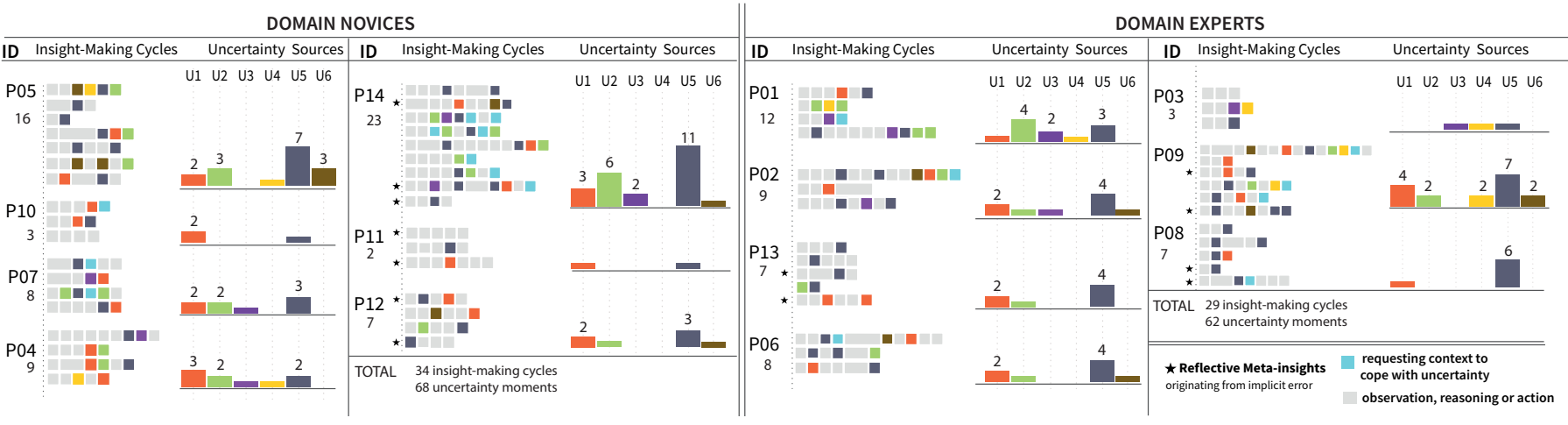

Fig. 3. Results from the insight analysis of the exploratory task. During the 63 insight-making cycles, we identified 130 moments of uncertainty which we then classified by source. Among these uncertainties we uncovered those originating from implicit errors among those (U1,U2,U3) as well as three other sources (U4,U5,U6). Overall, all but two insight cycles included such moments with the majority originating from uncertainties regarding the current hypothesis or observation (U5). An unexpected if less frequent source of uncertainty mentioned was uncertainty regarding the visualization development in itself (U4).

insight (i.e. 6 out of 130) these uncertainty types are special in that they were directly addressed to the interviewer or to those imagined making the visualization decisions. Domain experts expressed such concerns based on their past experiences: "I remember there were a lot of artifacts just labeled pre-historical. So how did you handle these situations?"(P9).

U5- Uncertainty in a Hypothesis or Observation describes unexpected encounters with new information as well as doubts regarding the observations or the participants' working hypotheses. These uncertainties fueled the insightmaking cycles and were often the starting points for establishing a trust calibration as described in the knowledgemaking model by Sacha et al. [9]. For instance P13 after stating that ".. there should have been more farms or hamlets.." explored the eras, methods, locations of those sites to understand the discrepancy between her expectation and the visualization reading. Being the most prominent source of expressing confidence (57 out of 130), these were present in all participants and most insights.

U6- Uncertainty from Background Knowledge are moments in which the participants reflected based on their personal relation to the data, including their lack of expertise. These were encountered regardless of the domain expertise, as domain experts expressed their lack of contextual or experiential information for specific data or sites, whereas domain novices felt uncertain about the accuracy of their reasoning. The presence of this uncertainty among highly trained domain experts is perhaps an indication of the critical stance archaeologists are trained towards, highlighting how first-hand experience with the data is necessary to feel confident to make claims "[..] but I do not know the [Location] material that well."(P9).

It should be noted that all these moments of uncertainty are highly interrelated and tend to build upon each other. To illustrate, the following excerpt narrates an insight cycle that followed an uncertainty of an initial observation (U5):

I am not a survey specialist (U6 - questioning background knowledge), but at least here it is intensive survey so there are some good indications that indeed it started in Hellenistic times, but very small I suppose (U1 - checking certainty of collection methods). But there are a lot of unknowns [...] and unknown means there is not enough data to do an estimate of the extent (U2 - reflecting on researcher interpretation). I was checking if I could see on how much survey data or sherds [the interpretation] is based on. (P2, insight 1$)$.

While all six sources of uncertainty (U1-U6) occurred during the exploration task, only a subset of these (U1, U2, U5) emerged when analyzing the transcriptions of the comparison task. This inconsistency comes to support calls for evaluating uncertainty in real data analysis-settings rather than through predefined and controlled tasks [47], as the same participants discussed more sources of uncertainty during open exploration.

\subsection{Externalizing Implicit Error in the Probe}

The dedicated probe features did not receive the same attention and their ability to inspire confidence or elicit discussions on the implicit errors varied. Specifically the probe seemed more suitable to address uncertainties originating from the data collection methods (U1) rather than those originating from the other two implicit errors (U2, U3).

Visualizing differing data collection methods. According to the logs, the majority of participants kept the data methods view visible (59\% of total log time). Participants described the data methods view as one of the most useful features and stressed the importance of having this general perspective of the data "You can really access [the information] in this view, because normally I did it in my mind, [..] but in this [view] we can really see the reliability of the data"(P9), "It is very useful, because in a lot of places we are comparing apples and oranges."(P6).

Including non-interpreted data. While participants brought up aspects of uncertainty due to researcher interpretation (U2), few participants mentioned this implicit error by using its purposefully designed feature, i.e. the noninterpreted data views. Many argued that "continuity and empirical data are already [encoded] in the interpretation"( $\mathrm{P} 4)$, when explaining why they did not switch to the other views.

Nevertheless, while the inclusion of non-interpreted data views was at times expressed as redundant, it permitted domain experts to be confident in the visualization overall: "I like the interpretation [..]. If I was trying to be more unbiased, maybe I would have liked the empirical data view." (P13). This 
ability of the non-interpreted data views to elicit overall confidence is further illustrated by how P3, a domain and data expert, was reluctant to engage in insight-making when starting from the interpretation view and instead was only listing the caveats of the classification. They argued they cannot take such a site interpretation for granted, but needed access to the underlying assumptions to trust this data. They only started looking for findings - rather than criticizing the data - when exploring the empirical, "less biased" view of the probe.

Representing assumptions of continuity. Compared to the empirical and the interpretation view, the continuity view appeared redundant as a dedicated insight-making representation as only four findings were made using exclusively this view. Accordingly, whereas domain novices often used the temporal axis as absolute time instead of chronology, this feature was almost completely ignored by the domain experts. Domain experts reasoned that assumptions about time and chronology were already intuitively integrated in their sense-making process "I understand the chronology and distances between the periods of time"(P6). In contrast, domain novices occasionally mentioned using the feature to show absolute time for its intended goal, i.e. to avoid bias (P14 and P10) "I wanted to have a better image of how time was divided in these periods"(P14).

\subsection{Coping with Implicit Error}

Participants were inclined to suggest or adopt particular strategies to overcome implicit error through the visualization probe. We adopt Boukhelifa et al.'s |10| classification of ignore, understand or minimize to describe these strategies which are also largely in agreement with the acknowledge, reduce and suppress mechanisms documented by Kale et al. [15]. Participants ignore -in our case uniquely actively ignore when they recognized the existence of uncertainty due to implicit error yet did not try or propose ways to understand it further; they understand when they tried to improve awareness of its origins for example by defining it further; and minimize when they proposed actions to alleviate such uncertainty to improve confidence in their findings. In our data, only around a quarter of the uncertainty moments were actively coped (33 from 130) as the coping strategies were often expressed with regards to the full insight rather than individual moments.

\subsubsection{Actively Ignoring and Understanding Uncertainty}

The majority of the participants without data experience used the dedicated visualization features to understand uncertainties originating from implicit error (in 6 of their 9 understood moments). These novice participants, understood the discrepancies regarding the data collection methods (U1) after becoming aware of them through the legend and only then continued to minimize them by suggesting to revisit the sites: "You can't really be sure of that. [..] Because here, it's just $10 \%$ coverage, so it would have potential to revisit that." (P7). Since the mentioned percentage does not exist in archaeological literature, we are confident that such a claim originated from our legend rather than one's background. On the contrary, although those with data experience also expressed similar uncertainties originating from the data collection methods (U1), they used their own experiences instead of the visualization to understand them: "I know there are a lot of Ottoman stuff, we just don't collect them." (P12).

Depending on their data experience, most domain novices could not handle implicit errors further $(85 \%$ of moments were not coped). This discrepancy between simply being aware of an uncertainty versus also having the ability to cope with it was especially evident in the comparison task: "Yeah, I would certainly agree with this (statement of comparability), [..] the only big difference is that one was surveyed different than the other, but I think for the rest it's quite the same." (P5). Domain novices with no first-hand experience of surveying (P4, P5, P7, P14), simply acknowledged or identified method mismatches (in 8 of the 10 of U1 moments). However, those with data knowledge (P10, P11, P12), having conducted field-work, were able to shift from acknowledging the uncertainty to suggesting how to minimize it "I would advise them to revisit [Location] and maybe grid it and to walk over it again." (P11, task 2).

\subsubsection{Adding Contextual Information}

Participants suggested two strategies to minimize uncertainties which we describe as depth and breadth to denote the tendency to uncover more detail of the existing data source versus searching for new information sources all together.

Depth Participants looked for ways to retrieve abstracted information from the visualization to become more confident in their insights (9 instances). This information may exist in various in-between manifestations of the data handling (e.g. the notebooks or archive reports) or even only in the original physical finds. For example, they wanted to know when a site was revisited and by whom to become more confident in the data collection methods (U1). Similarly, to be more confident about the interpretation (U2) they wanted to know the amount and spatial distribution of the collected finds, as well as the pottery sherd types and appearance: "I was checking if I could see on how much survey data or sherds it is based, because [..] if they are only two sherds, that is meaningless but if they are 2000 then we can actually say something."(P2).

Breadth Participants also wanted new information (breadth) that could help alleviate epistemological uncertainties in a forensic manner (8 instances). For example, they wished for additional topographical data of the investigated regions as well as research agendas to be more certain regarding the data collection methods (U1). Similarly, to alleviate uncertainties originating from the interpretation (U2), they inquired about topographical data, functional material types (i.e. did the artifacts include cooking ware or production materials) as well as the wider sociopolitical happenings of the time. "It's not even sure that in Byzantine times they are still belonging to the same political sphere [..] so the explanation might be completely different."( $\mathrm{P} 2$, task 2).

While this claim cannot be generalized, our data at least suggest that participants with some data experience who conversely had first-hand experience with the deployed methodologies, felt the need for more contextual breadth in order to increase their confidence (5 of the 6 instances where data experienced participants requested contextual information), whereas domain experts without data experi- 
ence expressed the need for more contextual depth (8 of 11 instances).

\subsubsection{Socially Grounding Uncertainty}

We observed how data analysis is a socially embedded process, i.e. it is performed as part of a community and therefore actions within it are impacted both by relations to others as well as one's own lived experiences.

Others. Participants at times coped with uncertainties by relating with the researchers who collected or classified the data rather than the data itself. Namely, social relations served to actively ignore uncertainties directly: "It might be logical [..] because [it is] based on the finds and the interpretations of the team [..]."(P4). This transfer of confidence from the data to the researchers hides an extra layer of situational knowledge that is established through social relations ".. it should be quite OK cause it is the work of [person's name] so its intensive survey [..." (P2, implying they know that person only intensively surveys and therefore can be trusted).

Oneself. Participants with data experience often also narrated first-hand memories of their personal experiences of surveying the data in order to reason about or ignore an emerging uncertainty. "[site name] was unique since they were installing a sprinkler system [...] and perhaps [this] contributes to the fact that we can label it now as the site with the longest occupation."(P8).

\section{Discussion}

We described how the visualization of implicit error in an archaeological context influenced confidence in the sensemaking of a variety of participant types. We now synthesize these rich and contextually grounded observations into critical considerations.

\subsection{How Users Relate to Implicit Error}

A user's background in archaeological data determines how she uses and benefits from the externalization of implicit error. We observed how data novices acknowledged implicit error through primary (e.g. the temporal matrix) and secondary (e.g. the legend) sections of the visualization. Such novices would probably unknowingly have ignored these implicit errors for the same task, as common analytical tools in archaeological research lack these kinds of features. Domain experts however, have already developed awareness of the implicit errors in the data as part of their professional development of data vision - i.e. the discretion around 'why, how, and when to improvise around established methods and tools in the wake of empirical diversity' [61]. As such, the visualization of implicit error can be exploited to teach domain novices to reflect upon the confidence of their insights and as a tool towards developing their own professional data vision.

Our archaeological domain and data experts 'trusted' the visualization that includes implicit error features, even if they did not actively use all features during their sensemaking. We observed the expert participants who used both our initial prototype and the redesigned visualization probe being less critical when engaging with insight-making with the latter. We believe this trust and lack of criticality partially originates from estimating the perceived effort that is required from the visualization developer to recognize and then conscientiously account for implicit errors in the visualization. This effort not only testifies to the developer's commitment to engage in a transdisciplinary way [50], it also shows respect to the idiosyncrasies and mannerisms of the targeted domain. The reluctance to embrace visual analytical tools in the humanities is now well documented [31], [44] and is commonly attributed to visualization's reductionist handling of information that does not comply with the practices and mental models in the humanities. Working closely with humanists to understand the implicit errors in their data and then externalizing them in the resulting visualizations can perhaps help regain the trust of the humanists in visual analytic systems.

Takeaway: Consider to visualize implicit error as a way to engage domain experts or others holding a critical data stance. Working closely with experts to get a deeper appreciation of how implicit errors influence their interpretations can help establish rapport in the process, help them engage with the visualization tool and perhaps even add confidence in the findings gained from it.

Archaeologists tend to ignore implicit error when they judge the data by its social context. Hidden behind much of the data in archaeology, and probably the humanities in general, lays a tacit relation to the data as well as the personal relationships with the people who gathered, classified and maintained that data. In practice, this means that a user's own (i.e. first-hand) or socially embedded (i.e. colleagues') experience is mentally linked to how they will assess the impact of various implicit errors. While trust in scientific data as a socially embedded process has already been empirically studied in corporate data science projects [62], in military intelligence analysis [63] and in larger distributed data consortia [64], we believe it is understudied in the context of visualizations in general, and of visualizations for humanistic research more specifically. In data visualization literature, the personal relationship to data is most commonly considered as a valuable asset in casual usage scenarios such as when individuals are invited to make data visualizations for themselves [65], when representing personal data [66], [67], or when looking for personal relations to drive them into data |68|. Our work adds to this knowledge by demonstrating how even in a professional setting, social factors can potentially become a unique and strong coping mechanism for uncertainties originating from implicit error.

Takeaway: Consider leveraging the social relationships of the people and organizations who handled the data, in order to empower users to cope with the implicit errors they encounter. While it may be challenging to represent such situational aspects directly, the visualization can include simple cues (like labels, links, explanations) that allow users to relate to experiences or people they deem trustworthy. Naturally, visualization designers may want to purposefully design against the social context around the data for example because it could introduce a social bias that clouds the findings or even because it could raise privacy concerns. 


\subsection{Implicit Error in Visualization and Coping with it}

The visualization of implicit error can be leveraged to spark reflective meta-insights. By externalizing the data collection methods alongside the data, domain experts observed the impact of those methods on their hypotheses and they even used these to plan future fieldwork strategies. We define reflective meta-insights as insights that originate directly from the externalized implicit errors rather than the perceived main data dimensions. Such meta-insights drive reflection on domain practices and methodologies more broadly. Parallel to how our inclusion of implicit errors brought about meta-insights, analytic decision graphs [17] that represent decisions made throughout a research cycle aim to elicit similar reflection on research choices. We argue such visualization solutions address the call to 'exploit' uncertainties rather than only cope with them [10]. Namely, even if authors avoid representing uncertainty in visualizations because they expect reader to encounter difficulties in interpreting it [35], our results propose that valuable scenarios of use exist in which the explicit sense-making around uncertainty could be the aim in and by itself.

Takeaway: Consider treating implicit error as an inspirational signal rather than noise. While its benefits may be indirect, it can support users to leverage uncertainty and ultimately influence domain practices that contribute to such error generation in the first place.

Although implicit error was not fully 'minimized' through visualization alone, archaeologists expected more contextual depth as well as breadth to cope with it. In our case study, even the most experienced domain experts questioned strong observations when they were grounded in data they had no first-hand interaction with. Moreover, even when making the same insight, participants diverged on the impact of its implicit errors and how to cope with them. Such approaches to uncertainty demonstrate why it is hard for representational abstractions to truly alleviate implicit error in the humanities. Their epistemic nature always adds a subjective factor, leaving it open to interpretation.

Our participants required additional contextual information to minimize uncertainty originating from implicit error. They did so either by wanting to reach in the depth of the data, via links to the original artifacts or their various in-between manifestations or alternatively by reaching for breadth via additional primary data sources, such as topographical data. Overall, this expectation to reach even deeper within and outside of the source data confirms how digital humanities research designs its visualizations to maintain a direct relation to their original materials [39], [41], [42], [43], [69]. We believe that while these two uncertainty minimization directions originated from a case study of archaeologists, they are comparable to broader analysis strategies. As such, we propose they can be further examined as conceptual and practical lenses through which to evaluate including uncertainty features in visual analysis.

Takeaway: Consider what constitutes depth and breadth of the data in the specific collaboration settings as these can be leveraged to further support confident sense-making. The visualization designer can then selectively include aspects commonly requested for contextualization while taking care not to overwhelm the visualization.

\subsection{Uncovering Implicit Errors in Domain Data}

Even though we first noted aspects of these implicit errors during early conversations with the domain experts, their relevance only became obvious after being able to appreciate broader archaeological practice. Specifically, we found that implicit errors were better articulated only after altering our collaborator's existing practices. In our case that meant introducing them to visualization as a means for analysis and exploration rather than uniquely a communication tool.

Takeaway. We suggest researchers to be sensitive to implicit errors during the early stages of visualization development and deploy collaborative activities, such as dedicated creative opportunity workshops (CVO) |70| to uncover how to address them. Such collaborative activities may benefit from using prototypes that bring domain experts out of their immediate comfort zone with regards to their data and thus encourage them to more explicitly discuss tacit aspects of their practices.

\subsection{Limitations}

Through providing abundant information, being reflective of archaeological practices and actively incorporating participant feedback, even in the form of co-authorship in this manuscript, we tried to be rigorous and transparent towards our own subjectivity and decision-making [13]. Nevertheless, as a long-standing, situated collaboration it is possible that other researchers would have approached such implicit errors differently thus altering their impact on the sense-making process. Our findings are based on a stratified convenience sample that is not enough to be directly generalizable to other disciplinary settings or even fully representative of all archaeological research. Finally, we also realize that the generation of insights and the uncertainty experienced during that process is based on a complex cognitive process that can only be partially captured - and even less quantified - through real, contextualized tasks. Accordingly this work should be considered in its exploratory nature and for its ability to inform future work.

\section{CONCLUSION}

This study examined the impact of externalizing implicit error on sense-making within an archaeological project. We identified three implicit errors that prevented our collaborators from confidently engaging in exploratory visual analysis. We designed and deployed a probe that visualized the sources of these errors and collected rich qualitative and quantitative data on archaeologists' uncertainty while using it. We believe our findings inform future visualizations to more explicitly handle the complexity of uncertain data for a range of application domains and especially for other highly data-critical fields such as the digital humanities.

\section{ACKNOWLEDGMENTS}

The authors would like to thank the Sagalassos team for their support, as well as the reviewers for their constructive feedback. This research was supported by the KU Leuven Research Fund and the Research Foundation Flanders. 


\section{REFERENCES}

[1] R. Chapman and A. Wylie, Evidential reasoning in archaeology. London: Bloomsbury Academic, 2016.

[2] M. Llobera, "Archaeological Visualization: Towards an Archaeological Information Science (AISc)," Journal of Archaeological Method and Theory, vol. 18, no. 3, pp. 193-223, 2011.

[3] G. Lucas, Understanding the Archaeological Record. Cambridge, New York: Cambridge University Press, 2012.

[4] P. Miller and J. Richards, "The Good, the Bad, and the Downright Misleading: Archaeological Adoption of Computer Visualization," Computer Applications and Quantitative Methods in Archaeology, pp. 19-22, 1995.

[5] F. Windhager, S. Salisu, and E. Mayr, "Uncertainty of What and for Whom - And Does Anyone Care? Propositions for Cultural Collection Visualization," in Workshop on Visualization for the Digital Humanities (VIS4DH), 2019.

[6] F. Windhager, V. A. Filipov, S. Salisu, and E. Mayr, "Visualizing Uncertainty in Cultural Heritage Collections," in Eurographics Proceedings. The Eurographics Association, 2018.

[7] A. M. MacEachren, A. Robinson, S. Hopper, S. Gardner, R. Murray, M. Gahegan, and E. Hetzler, "Visualizing Geospatial Information Uncertainty: What We Know and What we Need to Know," Cartography and Geographic Information Science, vol. 32, no. 3, pp. 139-160, 2005.

[8] T. Zuk and S. Carpendale, "Visualization of Uncertainty and Reasoning," Lecture Notes in Computer Science (including subseries Lecture Notes in Artificial Intelligence and Lecture Notes in Bioinformatics), vol. 4569 LNCS, pp. 164-177, 2007.

[9] D. Sacha, H. Senaratne, B. C. Kwon, G. Ellis, and D. A. Keim, "The Role of Uncertainty, Awareness, and Trust in Visual Analytics," IEEE Transactions on Visualization and Computer Graphics, vol. 22, no. 1, pp. 240-249, 2016.

[10] N. Boukhelifa, M. E. Perrin, S. Huron, and J. Eagan, "How Data Workers Cope with Uncertainty: A Task Characterisation Study," Proceedings of the ACM Conference on Human Factors in Computing Systems (CHI), pp. 3645-3656, 2017.

[11] N. McCurdy, J. Gerdes, and M. Meyer, "A Framework for Externalizing Implicit Error Using Visualization," IEEE Transactions on Visualization and Computer Graphics, vol. 25, no. 1, pp. 925-935, 2019.

[12] A. M. MacEachren, "Visual Analytics and Uncertainty: It's Not About the Data," in EuroVis Workshop on Visual Analytics. The Eurographics Association, 2015.

[13] M. Meyer and J. Dykes, "Criteria for Rigor in Visualization Design Study," IEEE Transactions on Visualization and Computer Graphics, vol. 26, no. 1, pp. 87-97, 2019.

[14] H. Hutchinson, W. E. Mackay, B. Westerlund, B. B. Bederson, A. Druin, C. Plaisant, M. Beaudouin-Lafon, S. Conversy, H. Evans, N. Roussel, H. Hansen, N. Roussel, B. Eiderbäck, S. Lindquist, and Y. Sundblad, "Technology probes: Inspiring Design for and with Families," Proceedings of the ACM Conference on Human Factors in Computing Systems (CHI), no. 5, pp. 17-24, 2003.

[15] A. Kale, M. Kay, and J. Hullman, "Decision-Making Under Uncertainty in Research Synthesis," Proceedings of the ACM Conference on Human Factors in Computing Systems (CHI), pp. 1-14, 2019.

[16] M. Fernandes, L. Walls, S. Munson, J. Hullman, and M. Kay, "Uncertainty Displays Using Quantile Dotplots or CDFs Improve Transit Decision-Making," Proceedings of the ACM Conference on Human Factors in Computing Systems (CHI), 2018.

[17] Y. Liu, T. Althoff, and J. Heer, "Paths Explored, Paths Omitted, Paths Obscured: Decision Points \& Selective Reporting in End-toEnd Data Analysis," Proceedings of the ACM Conference on Human Factors in Computing Systems (CHI), pp. 1-14, 2020.

[18] L. M. K. Padilla, M. Powell, M. Kay, and J. Hullman, “Uncertain About Uncertainty: How Qualitative Expressions of Forecaster Confidence Impact Decision-Making With Uncertainty Visualizations," Frontiers in Psychology, vol. 11, no. January, 2021.

[19] Y. S. Kim, L. A. Walls, P. Krafft, and J. Hullman, "A Bayesian Cognition Approach to Improve Data Visualization," Proceedings of the ACM Conference on Human Factors in Computing Systems (CHI), pp. 1-14, 2019.

[20] M. Franke, R. Barczok, S. Koch, and D. Weltecke, "Confidence as First-class Attribute in Digital Humanities Data," in Workshop on Visualization for the Digital Humanities (VIS4DH), 2019.

[21] Z. L. Tormala and D. D. Rucker, "Attitude Certainty: A Review of Past Findings and Emerging Perspectives," Social and Personality Psychology Compass, vol. 1, no. 1, pp. 469-492, nov 2007.
[22] J. V. Petrocelli, Z. L. Tormala, and D. D. Rucker, "Unpacking Attitude Certainty: Attitude Clarity and Attitude Correctness." Journal of Personality and Social Psychology, vol. 92, no. 1, pp. 3041, jan 2007.

[23] A. R. White, "Human Expertise in the Interpretation of Remote Sensing Data: A Cognitive Task Analysis of Forest Disturbance Attribution," International Journal of Applied Earth Observation and Geoinformation, vol. 74, no. August 2018, pp. 37-44, feb 2019.

[24] A. Burdick, D. Johanna, P. Lunenfeld, T. Prenser, and J. Schnapp, "Humanities to Digital Humanities," in Digital_Humanities. MIT Press, 2012, p. 140.

[25] A. Galey and S. Ruecker, "How a Prototype Argues," Literary and Linguistic Computing, vol. 25, no. 4, pp. 405-424, 2010.

[26] J. J. Thomas and K. A. Cook, Illuminating the Path: The Research and Development Agenda for Visual Analytics. IEEE Computer Society, 2005.

[27] A. J. Schofield, Interpreting Artefact Scatters: Contributions to Ploughzone Archaeology. Oxford : Oxbow, 1991.

[28] U. U. Schäfer, "Uncertainty Visualization and Digital 3D Modeling in Archaeology. A Brief Introduction." International Journal for Digital Art History, no. 3, 2019.

[29] R. Therón, A. G. Losada, R. Santamaría, and A. Benito, “Toward supporting decision-making under uncertainty in digital humanities with progressive visualization," ACM International Conference Proceeding Series, pp. 826-832, 2018.

[30] G. S. McCall, Strategies for Quantitative Research: Archaeology by Numbers. Abingdon: Routledge, 2018.

[31] J. Drucker, "Humanities Approaches to Graphical Display," DHQ: Digital Humanities Quarterly, vol. 5, no. 1, 2011

[32] A. M. Maceachren, R. E. Roth, J. O'Brien, B. Li, D. Swingley, and M. Gahegan, "Visual Semiotics \& Uncertainty Visualization: An Empirical Study," IEEE Transactions on Visualization and Computer Graphics, vol. 18, no. 12, pp. 2496-2505, 2012.

[33] S. Deitrick and R. Edsall, "The Influence of Uncertainty Visualization on Decision Making: An Empirical Evaluation," Progress in Spatial Data Handling - 12th International Symposium on Spatial Data Handling, SDH 2006, pp. 719-738, 2006.

[34] C. Kinkeldey, A. M. MacEachren, and J. Schiewe, "How to Assess Visual Communication of Uncertainty? A Systematic Review of Geospatial Uncertainty Visualisation User Studies," Cartographic Journal, vol. 51, no. 4, pp. 372-386, 2014.

[35] J. Hullman, "Why Authors Don't Visualize Uncertainty," IEEE Transactions on Visualization and Computer Graphics, vol. 26, no. 1, pp. 130-139, 2020.

[36] M. Greis, E. Avci, A. Schmidt, and T. Machulla, "Increasing Users' Confidence in Uncertain Data by Aggregating Data from Multiple Sources," Proceedings of the ACM Conference on Human Factors in Computing Systems (CHI), pp. 828-840, 2017.

[37] J. Liu, N. Boukhelifa, and J. R. Eagan, "Understanding the Role of Alternatives in Data Analysis Practices," IEEE Transactions on Visualization and Computer Graphics, vol. 26, no. 1, pp. 66-76, 2020.

[38] U. Hinrichs, S. Forlini, and B. Moynihan, "Speculative Practices: Utilizing InfoVis to Explore Untapped Literary Collections," IEEE Transactions on Visualization and Computer Graphics, vol. 22, no. 1, pp. 429-438, 2016.

[39] F. Windhager, P. Federico, G. Schreder, K. Glinka, M. Dork S. Miksch, and E. Mayr, "Visualization of Cultural Heritage Collection Data: State of the Art and Future Challenges," IEEE Transactions on Visualization and Computer Graphics, vol. 25, no. 6, pp. 2311-2330, 2019.

[40] M. Whitelaw, "Generous Interfaces for Digital Cultural Collections," DHQ: Digital Humanities Quarterly, vol. 9, no. 1, 2015.

[41] L. Manovich, "What is visualisation?" Visual Studies, vol. 26, no. 1, pp. 36-49, 2011.

[42] H. Lamqaddam, A. Vande Moere, V. Vanden Abeele, K. Brosens, and K. Verbert, "Introducing Layers of Meaning (LoM): A Framework to Reduce Semantic Distance of Visualization In Humanistic Research," IEEE Transactions on Visualization and Computer Graphics, vol. 27, no. 2, pp. 1084-1094, 2021.

[43] F. Gortana, F. von Tenspolde, D. Guhlmann, and M. Dörk, “Off the Grid: Visualizing a Numismatic Collection as Dynamic Piles and Streams," Open Library of Humanities, vol. 4, no. 2, pp. 1-25, 2018.

[44] N. McCurdy, J. Lein, K. Coles, and M. Meyer, "Poemage: Visualizing the Sonic Topology of a Poem," IEEE Transactions on Visualization and Computer Graphics, vol. 22, no. 1, pp. 439-448, 2016. 
[45] S. Tak, A. Toet, and J. Van Erp, "The Perception of Visual Uncertainty Representation by Non-experts," IEEE Transactions on Visualization and Computer Graphics, vol. 20, no. 6, pp. 935-943, 2014.

[46] M. Kay, T. Kola, J. R. Hullman, and S. A. Munson, "When (ish) is My Bus? User-centered Visualizations of Uncertainty in Everyday, Mobile Predictive Systems," Proceedings of the ACM Conference on Human Factors in Computing Systems (CHI), pp. 5092-5103, 2016.

[47] J. Hullman, X. Qiao, M. Correll, A. Kale, and M. Kay, "In Pursuit of Error: A Survey of Uncertainty Visualization Evaluation," IEEE Transactions on Visualization and Computer Graphics, vol. 25, no. 1, pp. 903-913, 2019.

[48] A. Dasgupta, J. Y. Lee, R. Wilson, R. A. Lafrance, N. Cramer, K. Cook, and S. Payne, "Familiarity Vs Trust: A Comparative Study of Domain Scientists' Trust in Visual Analytics and Conventional Analysis Methods," IEEE Transactions on Visualization and Computer Graphics, vol. 23, no. 1, pp. 271-280, 2017.

[49] C. Kinkeldey, A. M. MacEachren, M. Riveiro, and J. Schiewe, "Evaluating the Effect of Visually Represented Geodata Uncertainty on Decision-making: Systematic Review, Lessons Learned, and Recommendations," Cartography and Geographic Information Science, vol. 44, no. 1, pp. 1-21, 2017.

[50] K. W. Hall, A. J. Bradley, U. Hinrichs, S. Huron, J. Wood, C. Collins, and S. Carpendale, "Design by Immersion: A Transdisciplinary Approach to Problem-Driven Visualizations," IEEE Transactions on Visualization and Computer Graphics, vol. 26, no. 1, pp. 109-118, 2019.

[51] Z. Liu, N. J. Nersessian, and J. T. Stasko, “Distributed Cognition as a Theoretical Framework for Information visualization," IEEE Transactions on Visualization and Computer Graphics, vol. 14, no. 6, pp. 1173-1180, 2008.

[52] C. Turkay, A. Slingsby, H. Hauser, J. Wood, and J. Dykes, “Attribute Signatures: Dynamic Visual Summaries for Analyzing Multivariate Geographical Data," IEEE Transactions on Visualization and Computer Graphics, vol. 20, no. 12, pp. 2033-2042, 2014.

[53] F. Moretti, Graphs, Maps, Trees: Abstract Models for a Literary History. Verso, 2005.

[54] E. Banning, Archaeological Survey. Springer US, 2002.

[55] R. Vandam, E. Kaptijn, R. Willet, and P. Willett, "The countryside Where are the people?" in Meanwhile in the Mountains: Sagalassos., J. Poblome, E. Torun, P. Talloen, and M. Waelkens, Eds. Istanbul: Yapı Kredi Yayınları, 2019, pp. 262-270.

[56] J. Poblome and P. Bes, "This is how we do it. Methodology of Pottery Processing at Sagalassos," in Rei Cretariae Romanae Fautorum. Acta, vol. 45, Biegert, Susanne. Dr. Rudolf Habelt GmbH; Bonn, 2018, pp. 731-740.

[57] T. Hogan, U. Hinrichs, and E. Hornecker, "The Elicitation Interview Technique: Capturing People's Experiences of Data Representations," IEEE Transactions on Visualization and Computer Graphics, vol. 22, no. 12, pp. 2579-2593, 2016.

[58] P. Saraiya, C. North, and K. Duca, "An Insight-Based Methodology for Evaluating Bioinformatics Visualizations," IEEE Transactions on Visualization and Computer Graphics, vol. 11, no. 4, pp. 443-456, 2005.

[59] D. Sacha, A. Stoffel, F. Stoffel, B. C. Kwon, G. Ellis, and D. A. Keim, "Knowledge Generation Model for Visual Analytics," IEEE Transactions on Visualization and Computer Graphics, vol. 20, no. 12, pp. 1604-1613, 2014.

[60] R. E. Boyatzis, Transforming Qualitative Information: Thematic Analysis and Code Development. London, UK: SAGE, 1998.

[61] S. Passi and S. J. Jackson, "Data Vision: Learning to See Through Algorithmic Abstraction," Proceedings of the ACM Conference on Computer Supported Cooperative Work and Social Computing (CSCW), pp. 2436-2447, 2017.

[62] - "Trust in Data Science: Collaboration, Translation, and Accountability in Corporate Data Science Projects." Proceedings of the ACM Conference on Computer Supported Cooperative Work and Social Computing (CSCW), vol. 2, no. CSCW, pp. 1-28, Nov 2018.

[63] R. Walker, A. Slingsby, J. Dykes, K. Xu, J. Wood, P. H. Nguyen, D. Stephens, B. L. Wong, and Y. Zheng, "An Extensible Framework for Provenance in Human Terrain Visual Analytics," IEEE Transactions on Visualization and Computer Graphics, vol. 19, no. 12, pp. 2139-2148, 2013.

[64] A. L. Young and W. G. Lutters, "(Re)defining Land Change Science through Synthetic Research Practices," Proceedings of the ACM Conference on Computer Supported Cooperative Work and Social Computing (CSCW), pp. 431-442, 2015.
[65] A. Thudt, U. Hinrichs, S. Huron, and S. Carpendale, "SelfReflection and Personal Physicalization Construction," Proceedings of the ACM Conference on Human Factors in Computing Systems (CHI), pp. 1-13, 2018.

[66] R. Mortier, H. Haddadi, T. Henderson, D. McAuley, and J. Crowcroft, "Human-Data Interaction: The Human Face of the Data-Driven Society," Social Science Research Network, Rocherster, 2014.

[67] C. Elsden, A. Durrant, D. Chatting, D. Green, D. Kirk, and D. Abacus, "Abacus Datagraphy: A Speculative Enactment," Proceedings of the 3rd Biennial Research Through Design Conference, no. March, pp. 148-162, 2017.

[68] E. M. Peck, S. E. Ayuso, and O. El-Etr, "Data is Personal: Attitudes and Perceptions of Data Visualization in rural Pennsylvania," Proceedings of the ACM Conference on Human Factors in Computing Systems (CHI), pp. 1-12, 2019.

[69] K. Glinka, C. Pietsch, and M. Dörk, "Past Visions and Reconciling Views: Visualizing Time, Texture and Themes in Cultural Collections," Digital Humanities Quarterly, vol. 11, no. 1, 2017.

[70] E. Kerzner, S. Goodwin, J. Dykes, S. Jones, and M. Meyer, "A Framework for Creative Visualization-Opportunities Workshops," IEEE Transactions on Visualization and Computer Graphics, vol. 25, no. 1, pp. 748-758, 2018.

Georgia Panagiotidou is a $\mathrm{PhD}$ researcher at Research[x]Design and the Sagalassos Archaeological Research Project of the KU Leuven. Her work examines how to make visualization more inclusive by understanding how people handle data issues such as biases, uncertainties and frictions. She approaches data visualization both as a process and an outcome and therefore adheres to co-design practices.

Ralf Vandam is a senior postdoctoral fellow of the Research Foundation Flanders (FWO) at the Sagalassos Archaeological Research Project of the KU Leuven and a part-time professor of Archaeology in the Department of Art Studies and Archaeology at the Vrije Universiteit Brussel. $\mathrm{He}$ is a landscape archaeologist with a special focus on humanenvironment interactions in the past.

Jeroen Poblome is a professor of classical archaeology at KU Leuven. $\mathrm{He}$ directs the ICRATES Project (archaeologydataservice.ac.uk), the Sagalassos Archaeological Research Project (sagalassos.be) and the KU Leuven Institute for Cultural Heritage. He tries to publish widely, mainly through the lens of the Sagalassos Project, Jeroen has worked on questions of settlement formation and decline, production and consumption patterns, as well as long-term development of socio-ecological systems and resilience of regional communities in the face of change. Social relevance plays an increasing role in his interdisciplinary research design, mainly aimed at the current heritage community of Ağlasun, Turkey.

Andrew Vande Moere is a Professor in Design Informatics at the Department of Architecture of KU Leuven university in Belgium, where he co-directs the Research[x]Design group. His research explores the designerly opportunities of emerging technologies. 